\title{
Sensitive and Rapid RP-HPLC Quantification of Zoledronic Acid in a Hydroxyapatite-based Nanoparticles
}

\author{
D. K. KHAJURIA* AND REMA RAZDAN \\ Department of Pharmacology, Al-Ameen College of Pharmacy, Bengaluru-560 027, India
}

Khajuria and Razdan: RP-HPLC Determination of Zoledronic Acid in Nanoparticles

\begin{abstract}
The aim of this study was to develop and validate a rapid and sensitive reverse-phase high performance liquid chromatography method for the quantitative determination of zoledronic acid in nanoparticles. The mobile phase was a mixture of methanol $(30 \%)$ and $7 \mathrm{mM}$ tetra butyl ammonium hydrogen sulphate, $2 \mathrm{mM}$ di-sodium hydrogen orthophosphate and sulphate and $8 \mathrm{mM}$ di-potassium hydrogen orthophosphate (70\%), pH adjusted to 3 with trifluoroacetic acid. C18 column was used as a stationary phase. The flow rate was maintained at $1 \mathrm{ml} / \mathrm{min}$. The detection of the constituents was done at $215 \mathrm{~nm}$ using an ultraviolet detector. Method validation was executed according to the International Conference on Harmonisation guidelines. The retention time of zoledronic acid was $3.27 \pm 0.03 \mathrm{~min}(\mathrm{n}=6)$. The assay was linear over the concentration range of $200-800 \mu \mathrm{g} / \mathrm{ml}$. The proposed method was sensitive with the limit of detection and limit of quantification values equal to 200 and $800 \mu \mathrm{g} / \mathrm{ml}$, respectively. The method was accurate with percent mean recovery from $\mathbf{9 9 . 0 1} \pm \mathbf{1 . 3 1}$ to $100.80 \pm 0.094 \%$ and precise as percent relative standard deviation values were less than 1\%. The method was successfully applied for the estimation of zoledronic acid loading efficiency on hydroxyapatite nanoparticles and in vitro zoledronic acid release profile analysis in hydroxyapatite-based nanoparticles.
\end{abstract}

Key words: Zoledronic acid, nanomedicine, RP-HPLC, validation, ICH guidelines

Bisphosphonates are one of the most important bone resorption inhibitor drugs, which are used extensively in the management of skeletal diseases like osteoporosis, malignant hypercalcemia, bone metastasis and Paget's disease ${ }^{[1-3]}$. Bisphosphonates are the endogenous arbitrator of bone mineralization, capable of being adsorbed onto hydroxyapatite (HA) crystals in bone, hence providing bone stabilization and hindering its dissolution. Unlike pyrophosphate (P-O-P), bisphosphonates due to their P-C-P structure are more stable and resistant to acid hydrolysis ${ }^{[4]}$. Despite their therapeutic effects, bisphosphonates are currently used in various studies as suitable targeting agents in radiotherapy ${ }^{[4,5]}$. Previous studies from our laboratory and others have shown that bisphosphonates could be useful as drug bone targeted carriers for delivering various nanoparticle-based therapeutic interventions to bone tissues for skeletal-related diseases ${ }^{[6-10]}$. This approach prevents adverse effects such as toxicity due to non-specific tissue interactions, larger medicine dosages, higher costs, and even likely poor patient compliance towards therapy.
Zoledronic acid (ZOL) is a newer third generation bisphosphonate. It is a potent inhibitor of osteoclastic bone resorption. ZOL chemically is [1-hydroxy-2(1H-imidazol-1-yl)ethane-1,1-diyl]bis(phosphonic acid) hydrate (fig. 1) ${ }^{[1-3]}$. Among bisphosphonates, ZOL has a higher binding affinity for the bone HA. It was established that under conditions likely to stimulate bisphosphonate binding to the bone, ZOL has a superior binding affinity for bone HA crystals as compared to other bisphosphonates. Therefore, after intravenous injection, ZOL is swiftly cleared from the circulation and gets localized to the bone ${ }^{[7]}$.

Literature survey revealed several reverse phasehigh performance liquid chromatography (RP-HPLC)

This is an open access article distributed under the terms of the Creative Commons Attribution-NonCommercial-ShareAlike 3.0 License, which allows others to remix, tweak, and build upon the work non-commercially, as long as the author is credited and the new creations are licensed under the identical terms

Accepted 18 May 2017

Revised 17 January 2017

Received 13 October 2016

Indian J Pharm Sci 2017;79(4):553-558 


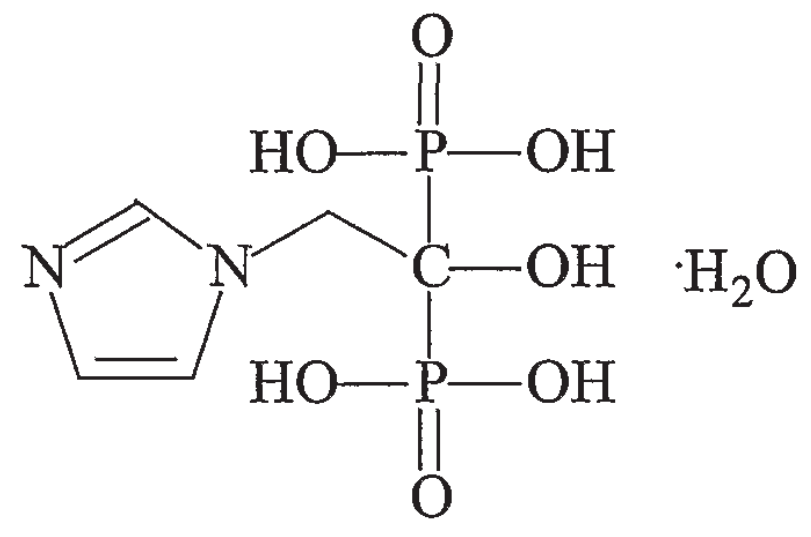

Fig. 1: Chemical structure of zoledronic acid

methods for determination of ZOL in injections and tablets ${ }^{[1-14]}$. But most of these methods are tedious, time-consuming, and require complex sample preparations. However, a simple and rapid quantitative HPLC method for estimation of ZOL in nanoparticlebased formulations has not yet been reported. Therefore, development of a suitable RP-HPLC analytical method for estimating ZOL in bone-targeted nanocarrier-based formulations appeared to be essential.

Development of a chromatographic method for the quantitative estimation of ZOL in nanoparticlebased formulations presented the following major difficulties, ZOL can chelate to metals and metal surfaces, generating chromatographic peak tailing; ZOL has no ultraviolet absorption or fluorescence, hence not readily detectable and as ZOL consisted of two phosphonate groups connected to a single carbon atom, it is poorly retained on non-polar stationary phase such as $\mathrm{C}_{18}$ or $\mathrm{C}_{8}$ column. For these reasons, ion-exchange liquid chromatographic methods are required for analysis of $\mathrm{ZOL}^{[11]}$. The main objective of this study was to develop a sensitive and rapid RP-HPLC method to assay ZOL in HA-based nano formulation for osteoporosis. The retention problem of ZOL has been successfully resolved by adding ion-pair reagent in the mobile phase. Our study appears to be the first to report a short run time of $3.2 \mathrm{~min}$ in the RP-HPLC method for ZOL, which facilitated analysis of several hundred samples per day. This RP-HPLC method offered significant advantages owing to its simplicity and rapidness for the detection of ZOL in the nanoparticle-based formulations.

\section{MATERIALS AND METHODS}

ZOL was obtained from Naprod Life Sciences Pvt. Ltd., Thane, Maharashtra, India. HPLC grade calcium nitrate tetrahydrate, phosphoric pentoxide, ethanol, sodium hydroxide, methanol, tetra butyl ammonium hydrogen sulphate, di-potassium hydrogen orthophosphate, trifluoroacetic acid and di-sodium hydrogen orthophosphate were purchased from Merck, Mumbai, India. High pure water was prepared by using Millipore Milli Q plus purification system.

\section{Preparation of bone targeted ZOL-loaded HA nanoparticles:}

ZOL-loaded HA nanoparticles were prepared according to our previously reported method ${ }^{[7]}$. Briefly, HA nanoparticles were synthesized first by sol-gel method in which calcium nitrate tetrahydrate and phosphoric pentoxide were used with the molar ratio of 10:3. Absolute ethanol was used as the solvent. After dissolving, the above precursors slowly transformed into a gel after $24 \mathrm{~h}$ under continuous stirring at ambient temperature. The formed gel was dried in an oven at $80^{\circ}$ in the air for $24 \mathrm{~h}$ and then sintered at $600^{\circ}$. Adsorption method was employed for the preparation of ZOL-loaded HA nanoparticle-based formulation ${ }^{[7]}$.

For estimation of ZOL loading on HA, $10 \mathrm{mg}$ weighed sample of dried nanoparticles was dissolved in $5 \mathrm{ml}$ of methanol. The sample was sonicated for $20 \mathrm{~min}$, made up to $10 \mathrm{ml}$ with methanol, and was filtered using a $0.2 \mu \mathrm{m}$ syringe filter (Axiva Sichem Biotech, New Delhi, India). ZOL content in the formulation was determined by RP-HPLC and drug loading efficiency was calculated ${ }^{[15]}$. For in vitro release studies, about $100 \mathrm{mg}$ of HA/ZOL nanoparticle-based drug formulation was transferred to a $100 \mathrm{ml}$ beaker containing $50 \mathrm{ml}$ of phosphate buffer saline (PBS) $\mathrm{pH}$ of 7.4 and continuously stirred at $100 \mathrm{rpm} 37^{\circ}$. Samples were withdrawn at various time intervals from the beaker and ZOL content was measured using the RP-HPLC method. An equal volume of PBS replaced the samples that were withdrawn.

\section{HPLC instrumentation and chromatographic conditions:}

The HPLC system consisted of a Shimadzu LC-20AD precision isocratic pump and an SPD-20A UV/Vis detector (Kyoto, Japan). Data collection and integration was accomplished utilizing LC Solutions chromatography Data System (Kyoto, Japan). A PHS-2C precision $\mathrm{pH}$ meter (Elico, LI 614, Hyderabad, India) equipped with a combination $\mathrm{pH}$ electrode was used for $\mathrm{pH}$ determination of the mobile phase. The chromatographic separation was achieved on a Phenomenex Luna C18 (2) analytical 
column $(250 \times 4.6 \mathrm{~mm}, 5 \mu \mathrm{m}$ particle size $)$ fitted with a Phenomenex analytical security Guard Cartridge System. The mobile phase consisted of a mixture of phosphate buffer that contained, 7 tetra butyl ammonium hydrogen sulphate, $2 \mathrm{mM}$ di-sodium hydrogen orthophosphate and sulphate and $8 \mathrm{mM}$ di-potassium hydrogen orthophosphate $(\mathrm{pH}$ of the mixture was adjusted to 3 with trifluoroacetic acid) and methanol $(70: 30, \mathrm{v} / \mathrm{v})$. The composition of the phosphate buffer was prepared according to the ZOL HPLC assay method previously reported by Rao et $a l .{ }^{[13]}$. The flow rate of the mobile phase was $1 \mathrm{ml} / \mathrm{min}$. The column temperature was maintained at $25^{\circ}$ and the wavelength was monitored at $215 \mathrm{~nm}$. The injection volume was $20 \mu$ l. The total run time for each LC run for the assay estimation was $8 \mathrm{~min}$.

\section{Linearity and range:}

A stock solution of ZOL $(10 \mathrm{mg} / \mathrm{ml})$ was prepared in methanol. The solutions were filtered through a $0.45 \mu \mathrm{m}$ nylon filter. Successive volumes of the standard solution were injected and chromatograms were recorded until the reproducibility of the peak area was satisfactory. Several standard solutions in the range of $200-800 \mu \mathrm{g} / \mathrm{ml}$ were used to define linear range of ZOL. The data of peak area versus drug concentration were treated by linear least square regression.

\section{Accuracy as recovery:}

Accuracy was determined by recovery study using standard addition method. The pre-analysed samples were spiked with extra 50,100 , and $150 \%$ of the standard ZOL and the mixtures were analysed by the proposed method. The experiment was conducted in triplicate.

\section{Precision:}

The intraday (repeatability) and interday (intermediate precision) variations for the determination of ZOL was carried out at three concentration levels of 200, 400, and $800 \mu \mathrm{g} \times \mathrm{ml}^{-1}$. The determinations were carried out in triplicate.

\section{Robustness:}

Robustness was carried out by making very small changes in the analytical methodology at a single

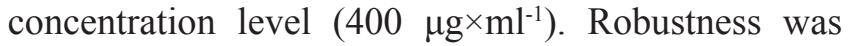
determined by making deliberate modifications in the mobile phase ratio, flow rate, and detection wavelength for analysis. The percentage of relative standard deviation (\%RSD) was also calculated to assess the robustness of the proposed method.

\section{Detection and quantification limits:}

The limit of detection (LOD) is the minimum amount of analyte in a specimen, which can be identified but not necessarily quantitated. The limit of quantification (LOQ) is the minimum amount of analyte in a specimen, which can be quantitatively determined with proper precision and accuracy. The LOD and LOQ were determined based on the method of signal-to-noise ratio (International Conference on Harmonization guidelines ICH-Q2 (R1) 2005) utilizing the following Eqns., $\mathrm{LOQ}=10 \sigma / \mathrm{S}, \mathrm{LOD}=3: 3 \sigma / \mathrm{S}$, where, $\sigma$ is the standard deviation of the intercept of the calibration plot and $\mathrm{S}$ is the slope of the calibration curve.

\section{RESULTS AND DISCUSSION}

Although limited RP-HPLC methods for the determination of ZOL have been reported ${ }^{[11-14]}$, none of them reported their specificity in quantifying ZOL in nanostructured delivery systems. No methods are available for ZOL quantification from nanoparticlebased formulations where a high specificity is required to overcome the probable interference of the excipients. The primary goal was to develop a sensitive and rapid RP-HPLC method that can assay ZOL. The sensitivity of RP-HPLC method that uses UV-detection depends on upon the proper selection of wavelength. An injection of $400 \mu \mathrm{g} / \mathrm{ml}$ of the standard solution of ZOL was made and the UV/Vis profile was recorded (200-400 nm). From the UV/Vis profile, a detection wavelength of $215 \mathrm{~nm}$ was selected, as the drug gave better peak response. The detection wavelength of $215 \mathrm{~nm}$ was selected and satisfactory precision was achieved. In this wavelength, ZOL could be determined with high sensitivity.

Satisfactory assay method of ZOL was achieved with a Phenomenex Luna C18 (2) analytical column $(250 \times 4.6 \mathrm{~mm}, 5 \mu \mathrm{m}$ particle size $)$ using a mixture of aqueous, 7 tetra butyl ammonium hydrogen sulphate, $2 \mathrm{mM}$ di-sodium hydrogen orthophosphate and sulphate and $8 \mathrm{mM}$ di-potassium hydrogen orthophosphate (pH3) and methanol (70:30, v/v). The column efficiency was found good even after making 500 injections. Optimization of chromatographic conditions, which is based on reasonable retention time for $\mathrm{ZOL}$ and excellent peak shape of ZOL was achieved initially. ZOL has multiple $\mathrm{pKa}$ values due to the presence of the phosphate moiety attached to the single carbon atom. In IEC, selection of most appropriate buffered 
mobile phase (as counter ion), its concentration and $\mathrm{pH}$ would significantly affect the extent of ionization of compounds and, hence, their retention times. For this purpose, the buffer concentration was kept as low as possible to avoid competition of the counter ions with the retention of sample ions. The addition of an organic solvent to the mobile phase resulted in the decreased retention just as in case of reversedphase chromatography. Also, tetra butyl ammonium hydrogen sulphate was utilized as the ion-pair reagent. The optimized method developed resulted in the elution of ZOL at 3.27 \pm 0.03 . Fig. 2 showed the chromatogram of pure ZOL.

The ZOL-HA nanoparticle solution did not show any changes in the peak at the retention time of pure ZOL. This indicates that the excipients used in the nanomedicine product do not interfere in estimation ZOL. Hence, the specificity for ZOL peak under the selected chromatographic conditions was proved. The ZOL peak identity was proved by comparing the retention times of pure ZOL with the HA-ZOL nanomedicine, as shown in fig. 2.

Several standard solutions of ZOL were used to define a linear range. The calibration curve was linear in the range of $200-800 \mu \mathrm{g} / \mathrm{ml}$. A good linearity was established by a correlation coefficient (R2) value of $0.9994 \pm 0.0001$ (Table 1). A correlation coefficient is a statistical parameter used to measure the degree or strength of the type of relationship, and here, a higher correlation coefficient value (close to 1.0) indicated a high level of linear relationship between the concentration of ZOL and the peak area. No significant differences were observed in the slopes of standard curves as indicated by the low \%RSD of 0.30 . Table 2 shows the linear regression data for the calibration curve of ZOL.

Accuracy was examined by evaluating three concentrations of the standard ZOL solution previously analysed using standard addition technique. The
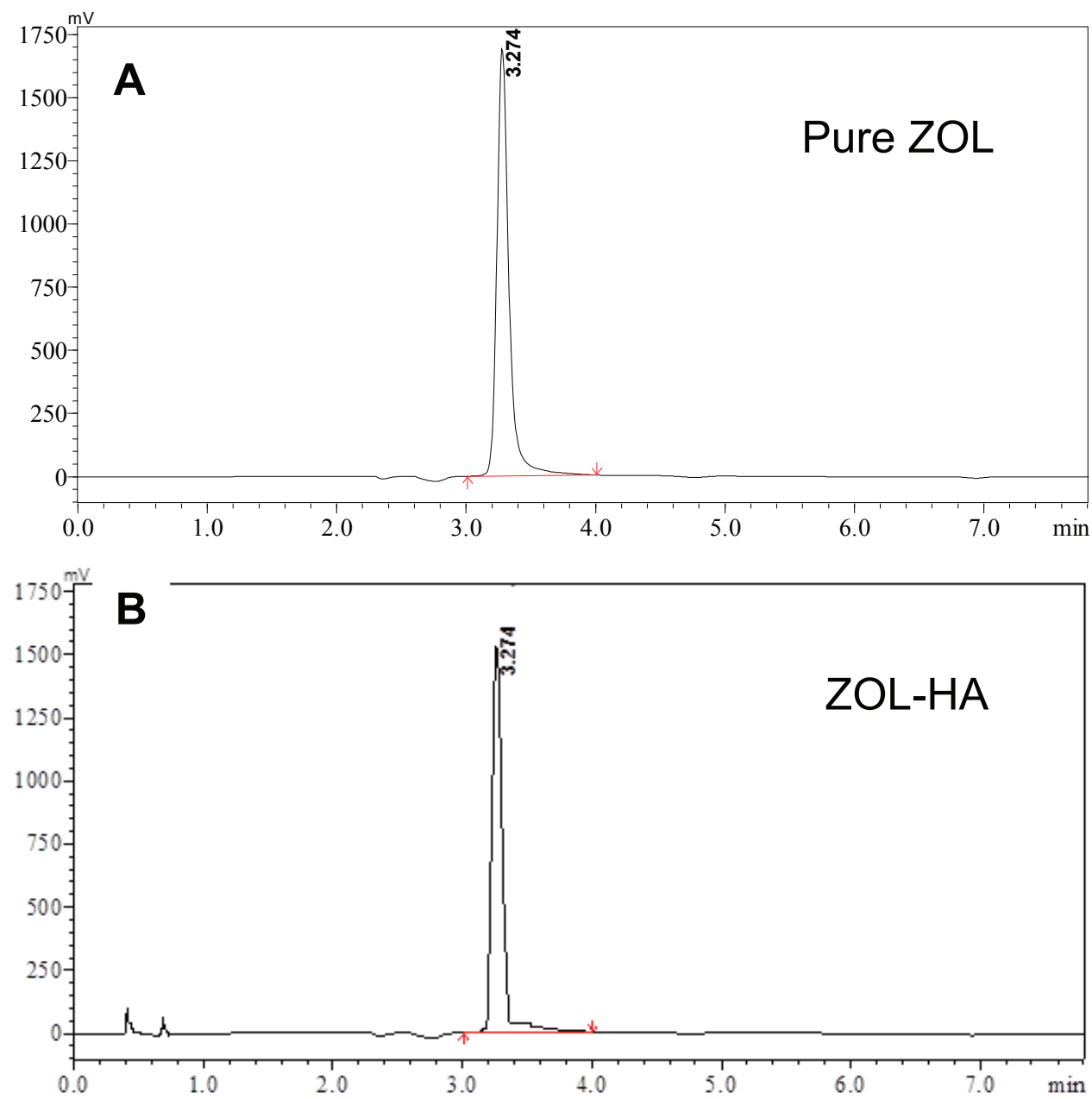

Fig. 2: Typical chromatogram ZOL and ZOL-HA nanoparticles Chromatograms of A. pure zoledronic acid (ZOL) and B. zoledronic acid-hydroxyapatite (ZOL-HA) nanoparticles 
recovery study was carried out to evaluate the accuracy of the proposed method to estimate ZOL. The standard addition technique was performed by adding 50, 100, and $150 \%$ of ZOL concentration in the sample. The percentage recoveries of the three ZOL concentrations were found to be $99.01 \pm 1.31$ to $100.80 \pm 0.094$, which indicated high accuracy. Percentage recovery and \%RSD values are shown in Table 2. The mean percentage recovery values, close to $100 \%$, and their low $\%$ RSD values indicated a high accuracy of the analytical method.

The repeatability of the developed RP-HPLC method, by intraday assay, was expressed in the terms of $\% \mathrm{RSD}$, and the results (Table 3 ) demonstrated the repeatability of the method. The inter day variation of $\mathrm{ZOL}$ at three different concentration levels of 200, 400, and $800 \mu \mathrm{g} \mathrm{ml}^{-1}$ establishes the intermediate precision of the

TABLE 1: LINEAR REGRESSION DATA FOR THE CALIBRATION CURVE

\begin{tabular}{lcc}
\hline Parameters & Mean \pm SD & \%RSD \\
\hline Linearity range $(\mu \mathrm{g} / \mathrm{ml})$ & 200 to 800 & - \\
Correlation coefficient $\left(\mathrm{R}^{2}\right)$ & $0.9994 \pm 0.0001$ & 0.02 \\
Slope & $13619.24 \pm 13.98$ & 0.30 \\
Intercept & $10906.51 \pm 825.62$ & 1.02 \\
\hline
\end{tabular}

$(\mathrm{n}=3)$ method. The low values of \%RSD for repeatability and intermediate precision shows an excellent precision of the developed RP-HPLC method.

Robustness was investigated by introducing minor changes in the mobile phase ratio, flow rate, and detection wavelength for analysis. The standard deviation and \%RSD of peak area and retention time $\left(\mathrm{R}_{\mathrm{t}}\right)$ was calculated and shown in Table 4. The low values of $\%$ RSD show the robustness of the developed RP-HPLC method. The LOD and LOQ were determined as per the ICH Guidelines Q2 (R1) (2005) and were found to be 200 and $800 \mu \mathrm{g} \times \mathrm{ml}^{-1}$, respectively.

Fig. 3 shows the in vitro release of ZOL from HA/ZOL nanoparticles, which ranged between $85.28 \pm 4.86 \%$ and $69.82 \pm 4.84 \%$ depending on the HA-to-ZOL ratio used. HA-to-ZOL ratio of $0.75: 1$ showed the maximum in vitro release of $85.28 \pm 4.86 \%$ after $70 \mathrm{~min}$ of dissolution in PBS.

A sensitive and rapid RP-HPLC method was developed and validated for the quantitative analysis of $\mathrm{ZOL}$ in nanoparticle-based formulations. The method validation was performed according to the $\mathrm{ICH}$ guidelines. The developed method was demonstrated

TABLE 2: RECOVERY DATA FOR THE ACCURACY OF THE HPLC METHOD

\begin{tabular}{lccccc}
\hline $\begin{array}{l}\text { Excess ZOL } \\
\text { added }(\%)\end{array}$ & $\begin{array}{c}\text { Content of sample } \\
(\mu \mathrm{g} / \mathrm{ml})\end{array}$ & $\begin{array}{c}\text { Theoretical content of } \\
\text { spiked sample }(\mu \mathrm{g} / \mathrm{ml})\end{array}$ & $\begin{array}{c}\text { Estimated Content } \\
\pm S D(\mu \mathrm{g} / \mathrm{ml})\end{array}$ & Recovery \pm SD $(\%)$ & $\%$ RSD \\
\hline 50 & 400 & 450 & $451.32 \pm 1.53$ & $99.01 \pm 1.31$ & 1.15 \\
100 & 400 & 500 & $502.72 \pm 1.26$ & $101.09 \pm 0.78$ & 0.68 \\
150 & 400 & 550 & $551.29 \pm 1.83$ & $100.80 \pm 0.94$ & 0.71 \\
\hline
\end{tabular}

TABLE 3: REPEATABILITY AND INTERMEDIATE PRECISION OF HPLC METHOD

\begin{tabular}{|c|c|c|c|c|}
\hline Concentration of sample $(\mu \mathrm{g} / \mathrm{ml})$ & Mean peak area \pm SD & \%RSD* & Mean peak area \pm SD & \%RSD* \\
\hline 200 & $467291.0 \pm 4415.3$ & 0.62 & $467149.3 \pm 3698.1$ & 0.61 \\
\hline 400 & $934586.5 \pm 5215.7$ & 0.41 & $934299.8 \pm 4287.9$ & 0.37 \\
\hline 800 & $1869166.2 \pm 6423.5$ & 0.34 & $1868596.6 \pm 7423.5$ & 0.32 \\
\hline
\end{tabular}

*Each value is a mean of three observations

TABLE 4: ROBUSTNESS DATA OF THE DEVELOPED RP-HPLC METHOD

\begin{tabular}{|c|c|c|c|c|c|c|}
\hline \multirow{2}{*}{ Parameter } & \multicolumn{2}{|c|}{ Study condition } & \multirow{2}{*}{ Mean area \pm SD } & \multirow{2}{*}{$\% \mathrm{RSD}^{*}$ of area } & \multirow{2}{*}{$\mathrm{R}_{\mathrm{t}} \pm \mathrm{SD}$} & \multirow{2}{*}{$\% R S D^{*}$ of $R_{t}$} \\
\hline & Original & Used & & & & \\
\hline \multirow{3}{*}{$\begin{array}{l}\text { Mobile phase ratio } \\
\text { (Buffer/methanol) }\end{array}$} & $70: 30: 00$ & $68: 32: 00$ & $934178.2 \pm 4117.7$ & 0.41 & $3.279 \pm 0.031$ & 0.45 \\
\hline & & $70: 30: 00$ & & & & \\
\hline & & $72: 28: 00$ & & & & \\
\hline \multirow{3}{*}{$\begin{array}{l}\text { Flow rate } \\
(\mathrm{ml} / \mathrm{min})\end{array}$} & 1 & 0.9 & $934351.9 \pm 5824.2$ & 0.33 & $3.277 \pm 0.078$ & 0.42 \\
\hline & & 1 & & & & \\
\hline & & 1.1 & & & & \\
\hline \multirow{3}{*}{$\begin{array}{l}\text { Detection wavelength } \\
(\mathrm{nm})\end{array}$} & 215 & 213 & $934566.7 \pm 3859.9$ & 0.3 & $3.275 \pm 0.024$ & 0.3 \\
\hline & & 215 & & & & \\
\hline & & 217 & & & & \\
\hline
\end{tabular}

${ }^{*}$ Each value is a mean of three observations 


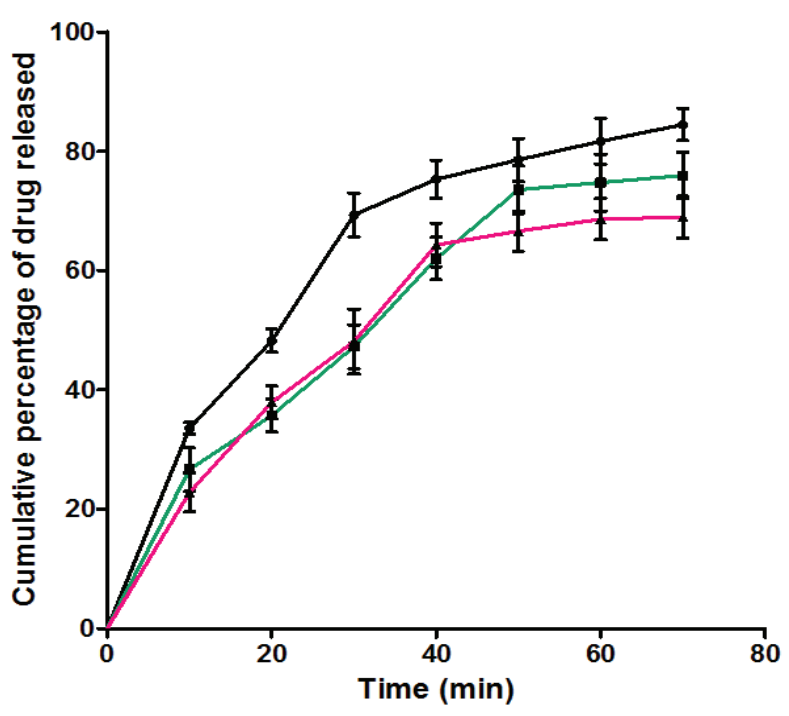

Fig. 3: Percent in vitro release of $\mathrm{ZOL}$ from $\mathrm{HA} / \mathrm{ZOL}$ nanoparticles

Mean \pm SD, $n=3$, - - HA-ZOL (0.75:1), - - HA-ZOL (0.50:1), $-\triangle-$ HA-ZOL $(0.25: 1)$

to be sensitive, rapid, accurate, and precise. The developed method could be successfully applied for the determination of the percentage ZOL loading, in vitro $\mathrm{ZOL}$ release profile and purification analysis of $\mathrm{ZOL}$ in nanoparticle-based drug formulations.

\section{Acknowledgements:}

Authors gratefully acknowledge financial support and research fellowship (File No: 45/35/2010-PHA/BMS) from Indian Council of Medical Research (ICMR), Govt. of India, New Delhi. Authors are also thankful to Mr. B. K Jain, Naprod Life Sciences, Maharashtra, India for providing gift sample of ZOL.

\section{Conflict of interest:}

The authors declare no conflict of interest.

\section{Financial support and sponsorship:}

This research was funded by the Indian Council of Medical Research (ICMR) (File No: 45/35/2010-PHA/ BMS), Govt. of India, New Delhi.

\section{REFERENCES}

1. Khajuria DK, Razdan R, Mahapatra DR. Drugs for the management of osteoporosis: a review. Rev Bras Reumatol 2011;51:365-82.
2. Khajuria DK, Disha C, Razdan R, Mahapatra DR. Comparative evaluation of zoledronic acid, alfacalcidol, and propranolol in pharmacological correction of experimental osteoporosis. Lat Am J Pharm 2013;32;968-76.

3. Khajuria DK, Disha C, Razdan R, Mahapatra DR, Vasireddi R. Prophylactic effects of propranolol versus standard therapy on a new model of disuse osteoporosis in rats. Sci Pharm 2014;82:357-74.

4. Paolino D, Licciardi M, Celia C, Giammona G, Fresta M, Cavallaro G. Bisphosphonate-polyaspartamide conjugates as bone targeted drug delivery systems. J Mater Chem B 2015;3:250-9.

5. Rudnick-Glick S, Corem-Salkmon E, Grinberg I, Yehuda I, Margel S. Near IR fluorescent conjugated poly(ethylene glycol)bisphosphonate nanoparticles for in vivo bone targeting in a young mouse model. J Nanobiotechnology 2015;13:1-8.

6. Khajuria DK, Disha C, Vasireddi R, Razdan R, Mahapatra DR. Risedronate/zinc-hydroxyapatite based nanomedicine for osteoporosis. Mater Sci Eng C Mater Biol Appl 2016;63:78-87.

7. Khajuria DK, Razdan R, Mahapatra DR. Development, in vitro and in vivo characterization of zoledronic acid functionalized hydroxyapatite nanoparticle based formulation for treatment of osteoporosis in animal model. Eur J Pharm Sci 2015;66:173-83.

8. Sahana H, Khajuria DK, Razdan R, Mahapatra DR, Bhat MR, Suresh $\mathrm{S}$, et al. Improvement in bone properties by risedronate adsorbed hydroxyapatite novel nanoparticle formulation in rat model of postmenopausal osteoporosis. J Biomed Nanotechnol 2012;9:1-9.

9. Swami A, Reagan MR, Basto P, Mishima Y, Kamaly N, Glavey $\mathrm{S}$, et al. Engineered nanomedicine for myeloma and bone microenvironment targeting. Proc Natl Acad Sci USA 2014;111:10287-92.

10. Rudnick-Glick S, Corem-Salkmon E, Grinberg I, GluzEMargel S. Doxorubicin-conjugated bisphosphonate nanoparticles for the therapy of osteosarcoma. JSM Nanotechnol Nanomed 2014;2:1022.

11. Jiang Y, Zhang XQ, Xu ZR. Analysis of zoledronic acid and its related substances by ion-pair RP-LC. Chromatographia 2004;60:405-9.

12. Legay F, Gauron S, Deckert F, Gosset G, Pfaar U, Ravera C, et al. Development and validation of a highly sensitive RIA for zoledronic acid, a new potent heterocyclic bisphosphonate, in human serum, plasma and urine. J Pharm Biomed Anal 2002;30:897-911.

13. Rao BM, Srinivasu MK, Rani CP, Kumar SS, Kumar PR, Chandrasekhar KB, et al. A validated stability indicating ionpair RP-LC method for zoledronic acid. J Pharm Biomed Anal 2005;39:781-90.

14. Zhang $\mathrm{X}$, Jiang $\mathrm{Y}, \mathrm{Xu} \mathrm{Z}$. Separation of zoledronic acid and its related substances by ion-pair reversed-phase high performance liquid chromatography. Chinese J Chromatogr 2004;22:428-30.

15. Gomes AJ, Faustino AS, Lunardi CN, Lunardi LO, Machado AE. Evaluation of nanoparticles loaded with benzopsoralen in rat peritoneal exudate cells. Int J Pharm 2007;32:153-60. 